\title{
A new route for high quality nanometric films of inorganic halide perovskites
}

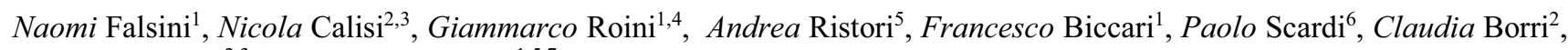
Stefano Caporali ${ }^{2,3}$, and Anna Vinattieri ${ }^{1,5,7}$

\footnotetext{
${ }^{1}$ Department of Physics and Astronomy, University of Florence, via G. Sansone 1, I-50019 Sesto Fiorentino (FI), Italy

${ }^{2}$ DIEF-Industrial Engineering Department, University of Florence, via S. Marta 3, I-50139 Florence, Italy

${ }^{3}$ INSTM-Interuniversity National Consortium for Material Science and Technology, via Giusti 9, I-50121 Florence, Italy

${ }^{4}$ Department of Information Engineering, University of Brescia, via Branze, 38, I-25123 Brescia, Italy

${ }^{5}$ LENS-European Laboratory for Non-Linear Spectroscopy, via Nello Carrara 1, I-50019 Sesto Fiorentino (FI), Italy

${ }^{6}$ Department of Civil, Environmental and Mechanical Engineering, University of Trento, via Mesiano 77, I-38123 Trento, Italy

${ }^{7}$ INFN-Firenze, via G. Sansone 1, I-50019 Sesto Fiorentino (FI), Italy
}

\begin{abstract}
Successful deposition of $\mathrm{CsPbX}_{3}(\mathrm{X}=\mathrm{Br}, \mathrm{Cl})$ thin films $(50-500 \mathrm{~nm})$ on several kind of substrates has been realized by Radio-Frequency Magnetron Sputtering. The technique allows for high homogeneity of the samples on large areas (several $\mathrm{cm}^{2}$ ) not only in terms of morphology but also referring to the optical properties. In particular, high resolution (in space, spectrum and time) photoluminescence studies in a wide temperature range $(10-300 \mathrm{~K})$ reveal that the low inhomogeneous broadening comes from a submicron size disorder while no significant contribution arises from a micrometric or even larger disorder. Given the relevance of inorganic halide perovskites for innovative optoelectronic devices, our results prove the scalability of this technique. Moreover, the successful deposition on several different substrates open the route for an easy integration in multi-layered structures.
\end{abstract}

\section{Introduction}

In the last decades fully inorganic halide perovskites have emerged as one of the most interesting class of semiconductor materials for applications in the field of energy harvesting, photonics and optoelectronics [1]. In particular, caesium-based halide perovskites are of extreme relevance for efficient incoherent (LED) and coherent (Laser) light emitters in the visible spectral region, being direct-band gap semiconductors, easily tunable by controlling the chemical composition. $\mathrm{CsPbCl}_{3}$ shows a blue emission of interest for white light generation, when coupled with a converting phosphor; $\mathrm{CsPBBr}_{3}$, having a bandgap at $2.3 \mathrm{eV}$, helps in overcoming the green gap problem of nitrides [2]. Moreover, white light generation can be obtained by multilayer structures of $\mathrm{CsPbI}_{3}, \mathrm{CsPbr}_{3}$ and $\mathrm{CsPbCl}_{3}$.

However, one of the main problems that limits the scalability of the material for innovative devices comes from the lack of homogeneity over large sample area (several $\mathrm{cm}^{2}$ ). In this work we demonstrate the first successful deposition of thin films of fully inorganic perovskite of $\mathrm{CsPbr}_{3}$ and $\mathrm{CsPbCl}_{3}$ by the RadioFrequency (RF) Magnetron Sputtering technique. In this way we succeeded in obtaining high quality samples with a very good uniformity and homogeneity concerning the morphological and optical properties.

\section{Results}

$\mathrm{CsPbr}_{3}$ and $\mathrm{CsPbCl}_{3}$ thin films were grown by $\mathrm{RF}$ Magnetron Sputtering on several substrates (soda lime glass - SLG, quartz, $\mathrm{TiO}_{2}$, Silicon, etc.) with thickness ranging between 50 and $500 \mathrm{~nm}[3,4]$.
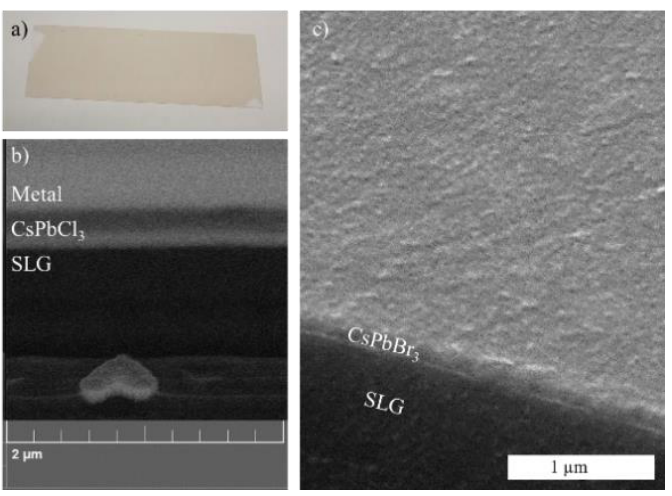

Fig. 1. a) Image of a $70 \mathrm{~nm} \mathrm{CsPbCl} 3$ film on quartz. b-c) SEM micrographs of a $150 \mathrm{~nm} \mathrm{CsPbCl}$ film and of a $140 \mathrm{~nm}$ $\mathrm{CsPbBr}_{3}$ film on glass.

\footnotetext{
* Corresponding author: vinattieri@fi.infn.it
} 
In Figure 1 a typical image of a $70 \mathrm{~nm} \mathrm{CsPbCl}$ film on quartz is shown along with two SEM micrographs of a $\mathrm{CsPbCl}_{3}$ and $\mathrm{CsPbBr}_{3}$ thin film. The samples appear to be highly uniform and homogeneous at the macro-scale (several $\mathrm{cm}^{2}$ ) and compact as from the SEM analysis. XPS measurements were performed, providing a substantial agreement with the expected stoichiometry, whereas XRD spectra clearly indicate the presence of the correct crystalline phase.

A detailed photoluminescence (PL) spectroscopy study was conducted at the macro and micro scale in a wide temperature range (10-300 K) to assess the origin of the inhomogeneous broadening and to quantify the PL quantum yield quenching. In figure $2 \mathrm{a}$ the PL spectrum at $10 \mathrm{~K}$ shows an inhomogeneous broadening less than 20 meV (FWHM); the comparison between macro-PL (excited area $\sim 10^{4} \mu \mathrm{m}^{2}$ ) and micro-PL (excited area $\sim 1$ $\mu \mathrm{m}^{2}$ ) spectra (Fig. 2b) indicate that the origin of the inhomogeneous broadening comes from a submicrometric scale disorder. A significant decrease of the PL intensity is observed starting from $\sim 150 \mathrm{~K}$ in both kind of samples and it originates from a reduced number of carriers and excitons that initially populate the radiative states [4].
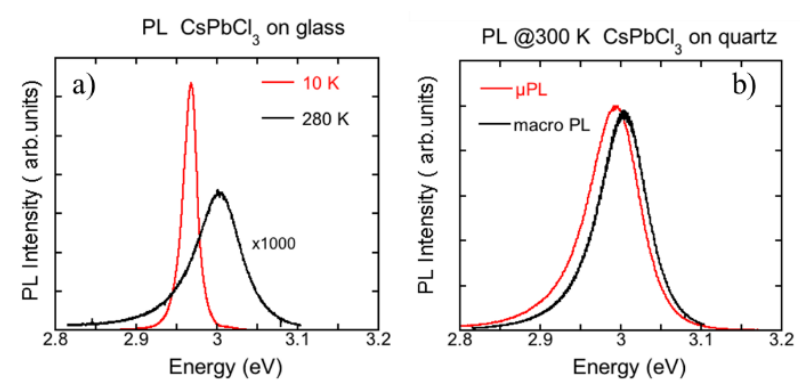

Fig. 2. a) $\mathrm{PL}$ spectra at $10 \mathrm{~K}$ and $280 \mathrm{~K}$ of a $\mathrm{CsPbCl}_{3}$ thin film on glass. b) Comparison between micro and macro-PL spectra at room temperature of $\mathrm{CsPbCl}_{3}$ thin film on quartz.

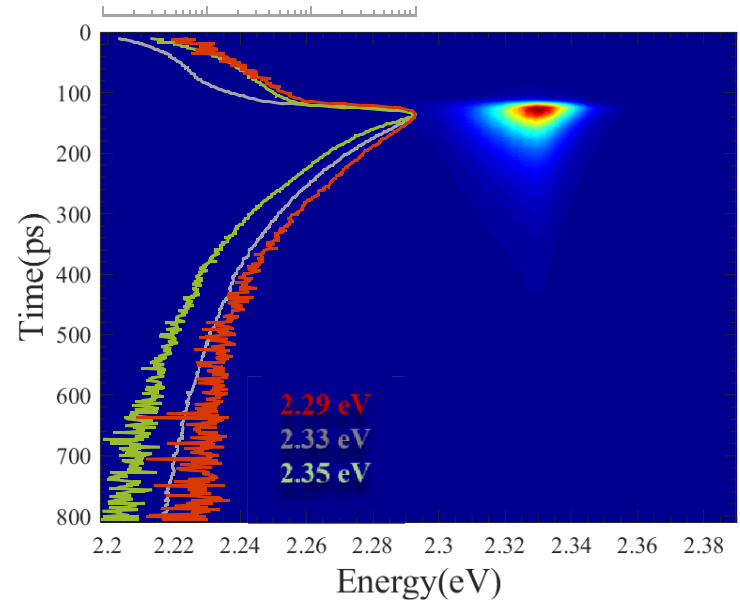

Fig. 3. Streak camera image of a $\mathrm{CsPbBr}_{3}$ film along with the normalized PL decays (Log scale) extracted from the image at three different energies: $2.35 \mathrm{eV}$ free carrier recombination, $2.33 \mathrm{eV}$ localized exciton emission, and $2.29 \mathrm{eV}$ low energy side due to deeper exciton localization.

Time-resolved (TR) spectra were acquired by a streak camera after excitation of the sample at $365 \mathrm{~nm}$ with 1.2 ps mode-locked laser (Figure 3).
Similar results are found for $\mathrm{CsPbBr}_{3}$ and $\mathrm{CsPbCl}_{3}$ : non exponential decays are commonly found in this class of materials with evidence of an exciton localization at low temperature and the presence of a thermalized high energy tail due to free carrier recombination [5].

Moreover, high satisfactory is the stability of PL signal in the whole sample area despite sample ageing. In fact, the loss of PL signal is of the order of few tens of percent after 2-3 months under ambient conditions, which, to our knowledge, is a considerable stability when compared with literature data.

\section{Conclusions}

We have demonstrated that high quality thin films of $\mathrm{CsPbX}_{3}(\mathrm{X}=\mathrm{Br}, \mathrm{Cl})$ can be realized by $\mathrm{RF}-$ Magnetron sputtering with state-of-the art homogeneity in terms of morphology and optical properties on sample areas as large as several square centimeters. In particular, this technique turns out to be highly promising for $\mathrm{CsPbCl}_{3}$ deposition because of limitations in realizing films by standard solution-based techniques.

\section{References}

1. Y. Kanemitsu, T. Handa, Jpn. J. Appl. Phys. 57, 1 (2018) https://doi.org/10.7567/JJAP.57.090101

2. K. Sim, T. Jun, J. Bang, H. Kamioka, J. Kim, H. Hiramatsu, H. Hosono, Appl. Phys. Rev., 6, 031402 (2019) https://doi.org/10.1063/1.5098871

3. C. Borri, N. Calisi, E. Galvanetto, N. Falsini, F. Biccari, A. Vinattieri, G. Cucinotta, S. Caporali, Nanomaterials, $\quad \mathbf{1 0}, \quad 60$ https://doi.org/10.3390/nano10010060

4. N. Falsini, N. Calisi, G. Roini, A. Ristori, F. Biccari, P. Scardi, S. Caporali, A. Vinattieri, Adv. Mater. (under submission)

5. F. Gabelloni, F. Biccari, N. Falsini, N. Calisi, S. Caporali, A. Vinattieri, Nanophotonics, 8, 1447 (2019) https://doi.org/10.1515/nanoph-2019-0013 